\title{
Magnetic Resonance Imaging of Changes in Muscle Tissues after Membrane Trauma
}

\author{
HANNE GISSEL ${ }^{a}$ FLORIN DESPA, JOHN COLLINS, DEVKUMAR MUSTAFI, \\ KATHERINE ROJAHN, GREG KARCZMAR, AND RAPHAEL LEE
}

Electrical Trauma Research Program, Biological Sciences Division, MC 6035, University of Chicago, Chicago, Illinois 60637, USA

${ }^{a}$ Department of Physiology and Biophysics, University of Aarhus, DK-8000 Århus C, Denmark

\begin{abstract}
A pure electroporation injury leads to cell membrane disruption and subsequent osmotic swelling of the tissue. The state of water in the injured area of a tissue is changed and differs from a healthy tissue. Magnetic resonance imaging (MRI), which is very sensitive to the quality of the interaction between mobile (water) protons and a restricted (protein) proton pool, is therefore a useful tool to characterize this injury. Here, we present a protocol designed to measure the difference between the values of the transverse magnetic relaxation time $\left(T_{2}\right)$ in MRIs of healthy and electrically injured tissue. In addition, we present a method to evaluate the two main contributions to the MRI contrast, the degree of structural alteration of the cellular components (including a major contribution from membrane pores), and edema. The approach is useful in assessing the level of damage that electric shocks produce in muscle tissues, in that edema will resolve in time whereas structural changes require active repair mechanisms.
\end{abstract}

KEYwORDS: electroporation; $\mathrm{T}_{2}$-MRI; electrical injury; edema

\section{INTRODUCTION}

It is well recognized that a high-current electrical shock often produces an irreversible damage of biological tissues, followed by necrosis. Damage may occur as a result of local heating to high temperature or as a direct result of breakdown of the cell membrane caused by electroporation. ${ }^{1-4}$ Heat may induce significant denaturation of the cellular components, whereas electroporation perturbs the delicate balance of ions and molecules between the intracellular and extracellular compartments. This will, among other things, lead to loss of muscle function, muscle $\mathrm{Ca}^{2+}$ overload, ${ }^{5-7}$ and most likely an increased production of reactive oxygen species (ROS). Increased production of ROS and muscle $\mathrm{Ca}^{2+}$ overload may in turn activate degradative processes intrinsic to the muscle cell, causing further muscle

Address for correspondence: Raphael C. Lee, Department of Surgery, MC 6035, The University of Chicago, 5841 South Maryland Avenue, Chicago, IL 60637. Voice: 773-702-6302; fax: 703-702-1634.

r-lee@uchicago.edu

Ann. N.Y. Acad. Sci. 1066: 272-285 (2005). () 2005 New York Academy of Sciences.

doi: 10.1196/annals.1363.024 


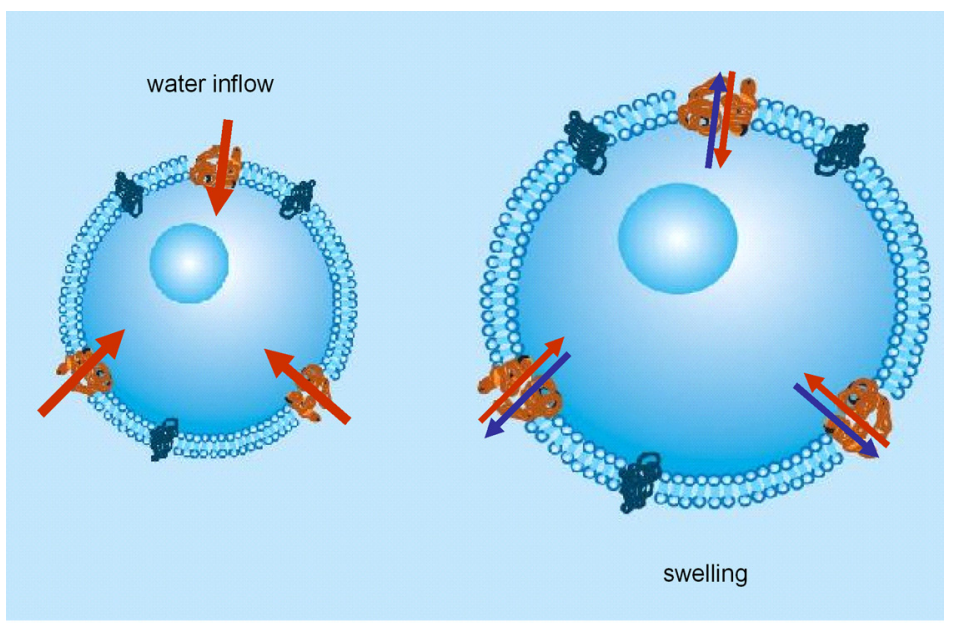

FIGURE 1. Electroporation of the plasma membrane leads to an osmotic water inflow, which in turn leads to cell swelling and increases the hydration of membrane compartments (fatty acids and proteins), which are usually separated from excess amount of water. At quasi-equilibrium, water inflow may be equal to the outer outflow (the reverse arrow).

damage ${ }^{8-10}$ Finally there may be development of edema due to osmotic water inflow into the muscle tissue.

In model studies of electrical trauma, the contribution to the tissue damage from heating and electroporation can be controlled by an appropriate choice of the electrical shock parameters. Sequences of high-voltage pulses in durations of milliseconds, separated by waiting times sufficiently long to allow for heat dissipation and thermal equilibration, may cause predominantly cell electroporation rather than heat (burn) damage. ${ }^{11}$ In contrast, high-frequency electric fields might lead primarily to thermal denaturation of the cellular components.

Membrane poration is a frequent injury mechanism in physical trauma of skeletal muscles. This has as a direct effect an alteration of the state of water in the tissue. First, disruption of cellular membranes increases the hydration of membrane compartments (fatty acids and proteins) which are usually separated from excess amounts of water (FIG. 1). Furthermore, structural modifications of cellular components change the dynamics of water molecules in the hydration layers. ${ }^{12}$ Secondly, swelling of the cell due to osmotic water inflow (edema) adds extra hydration layers to the cellular components (FIG. 1). These physiological changes affect the magnetic relaxation of the tissue water protons and are likely to be detected and measured by MRI. Water proton MR measurements of protein solutions has recently brought compelling evidence for the change of the MR signal due to protein unfolding. ${ }^{13-15}$ In addition, the magnetic relaxation dispersion curves revealed a change between long-lived and short-lived water proton populations during conformational changes of the protein. ${ }^{13}$ The physical basis of these observations is that protein unfolding increases the water-accessible surface area $(A S A)$, resulting in change of the number and dynamics of interface water molecules. To properly understand the origin of the 
change in water dynamics, we recall that a protein structure displays both binding (hydrophilic) and unbinding (hydrophobic) sites for water molecules and that, in the native state, the latter are buried inside the protein structure. Protein unfolding leads to the exposure of the hydrophobic residues to water, which induces a shift of the correlation decay time, that is, the time characterizing the motion of the water molecules in a specific vicinity with respect to the protein structure environment. ${ }^{13,14}$ This change in the dynamics of water is expected to occur at the interface with any cellular component subject to structural alterations, including the cell membrane, which seems to be primarily affected by the electric field.

Previous work demonstrated that electrical injury patterns can be visualized in MR images. ${ }^{16,17}$ It was shown that muscle subjected to electrical shock showed higher signal intensities in $\mathrm{T}_{2}$-weighted MRI images. These preliminary results had an immediate clinical impact and lay the ground for applying MRI as a diagnostic method in patients with electric injuries. ${ }^{18,19}$ Further investigations focused on edema localization and contrast agent distribution in a rat hind limb electrical injury model. ${ }^{20}$

In this chapter we present a protocol designed to measure the difference between the values of the $T_{2}$ relaxation time of healthy and electrically injured tissue. In addition, we present a method to evaluate the two main contributions to the MRI contrast, the degree of structural alteration of the cellular components (including a major contribution from membrane pores), and edema. The approach is useful in assessing the level of damage in muscle tissues produced by the electric shocks, as edema will resolve in time, whereas structural changes require active repair mechanisms.

\section{MEMBRANE DAMAGE BY ELECTROPRATION}

\section{The Animal Model}

Female Sprague-Dawley rats (weight $300 \pm 20$ g) were anesthetized using an intraperitoneal injection of a ketamine/xylazine combination (i.e., $90 \mathrm{mg} / \mathrm{kg}$ and $10 \mathrm{mg} / \mathrm{kg}$, respectively) dissolved in isotonic saline solution for anaesthesia induction. Additional anaesthetic was administered as needed to maintain a constant deep anaesthetic plane. Ketamine HCl Inj., USP (100 mg/mL, NDC 0856-2013-01) was from Fort Dodge Animal Health (Fort Dodge, IA 50501), and Xylazine sterile solution (20 mg/mL, NDC 11695-4400-1) from the Butler Company (Columbus, OH 43228). The experiments were done in accordance with the standards described in The Guide for the Care and Use of Laboratory Animals (NIH Publication No. 86-23, 1985, Department of Health and Human Services), under a protocol approved by the University of Chicago Institutional Animal Care and Use Committee.

All animals were fully anesthetized and medically stabilized before electrical shock. After the anesthetization took effect, catheters were surgically established and secured into the jugular vein and into the peritoneal cavity. A steady maintenance dose of ketamine/xylazine (i.e., $28 \mathrm{mg} / \mathrm{mL}$ and $0.20 \mathrm{mg} / \mathrm{mL}$, respectively at a rate of $0.35 \mathrm{~mL} / \mathrm{h}$ ) was administered i.p. throughout the procedure to maintain the anaesthetic plane below pain perception but above respiratory compromise. At the end of the experiment the animals were sacrificed by a Xylazine overdose $(0.4 \mathrm{~mL}$, $20 \mathrm{mg} / \mathrm{mL}$ ) administered intravenously. 


\section{The Electroporation Protocol}

The electrical conduction path was chosen between the ankle of the left leg and the base of the tail, and thus the electrical current travels along the whole leg of the rat. The skin at the ankle and at the base of the tail was abraded and wrapped in gauze soaked in Parker Electrode Gel, a salt-bridge conducting gel. Stainless-steel cuff electrodes were attached to the salt bridges and connected via high-voltage cables to the pulse generator. A discharge-type rectangular pulse generator (Dialog, Hannover, Germany) was used to generate the electrical shocks. The impedance of this circuit measured at $100 \mathrm{~Hz}$ was in the range of $1000 \pm 300 \mathrm{Ohms}$. All experiments were carried out in an electrically insulated chamber.

All rats received 12 shocks. Each shock consisted of a rectangular pulse of $2 \mathrm{kV}$, $\sim 2 \mathrm{~A}$ amplitude with duration of $4 \mathrm{~ms}$. There was a 10-second separation period between consecutive shocks to allow thermal relaxation following each pulse. It has
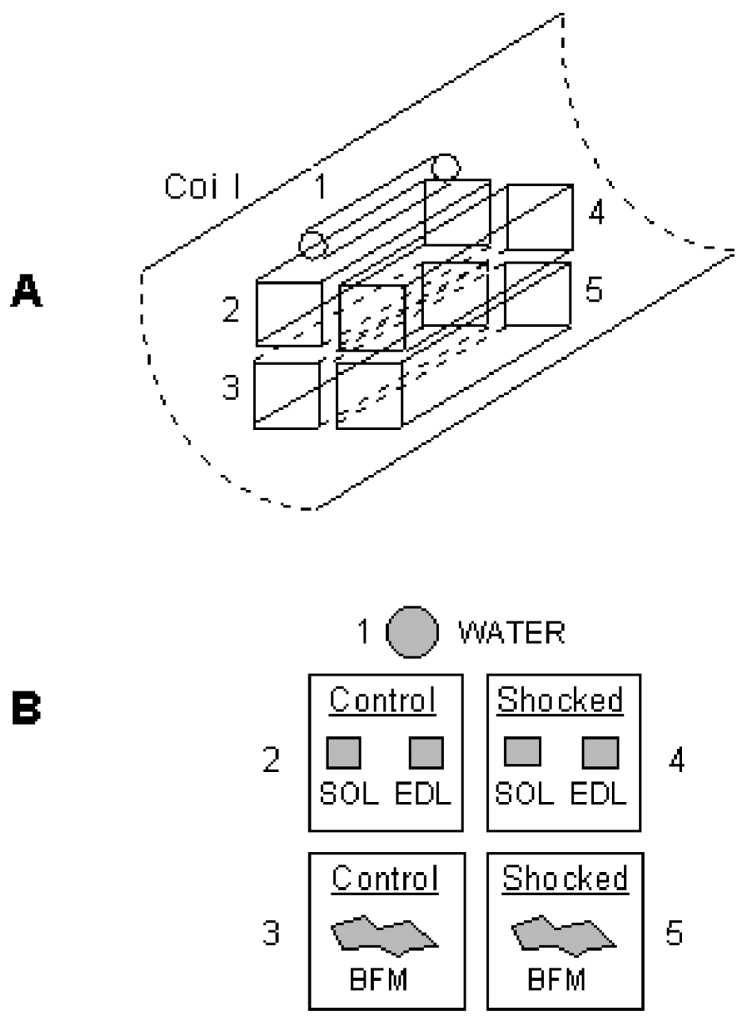

FIGURE 2. A: Illustration of the placing of the four 1-cm cuvettes (2-5) and the water phantom (1) in a 35-mm bird-caged coil for MR imaging. B: Cross-sectional view of the placing of the muscles in the cuvettes. Owing to their small size, SOL and EDL could be placed together in the same cuvette. Muscles from the control unshocked leg were placed in 2 and 3, whereas muscles from the shocked leg were placed in 4 and 5 (see text for details). 
been shown for this protocol that the temperature rise in the muscle tissue due to the electrical current pulses is less than $0.5^{\circ} \mathrm{C} .{ }^{11}$ The control animals were connected to the pulse generator for the same duration but did not receive electrical shocks.

All animals tolerated the anaesthetic induction and the electroporation injury. A 1-mL bolus of lactated Ringer's solution was administered intravenously immediately after shock and 1 hour post shock. The electroporated thigh muscles could be visually observed to swell over several hours.

Two series of experiments were conducted. In the first series (In vivo shock and ex vivo imaging) the rats were monitored and then sacrificed at 3 hours post shock. Intact extensor digitorum longus (EDL) and soleus (SOL) muscles were excised, together with a part of the biceps femoris muscle (BFM). Identical muscles isolated from the non-injured leg of the same rat served as controls. The muscles were immediately placed in sealed $1-\mathrm{cm}$ plastic cuvettes to avoid loss of moisture. A small water phantom was placed in the coil along with the muscles as a standard (FIG. 2A).

In the second series (in vivo shock and in vivo imaging) the rat was prepared for in vivo imaging immediately post shock. The shocked leg was imaged from approximately 1 hour post shock through 3 hours post shock. Images obtained separately from unshocked rats were used as controls.

\section{Magnetic Resonance Imaging}

Proton magnetic resonance images for $\mathrm{T}_{2}$ measurements were recorded using a Bruker scanner (Bruker Biospin, Billerica, MA) (200 MHz) equipped with a 4.7 T, $33-\mathrm{cm}$ internal diameter superconducting magnet and actively shielded magnetic field gradients. MRI measurements were acquired using a Bruker bird-caged coil with internal diameter of $35 \mathrm{~mm}$. Images were acquired with a standard multi-slice, multi-echo (MSME) spin echo Carr-Purcell-Meiboom-Gill (CPMG) sequence. The parameters for in vitro MRI measurements were as follows: TR, $2000 \mathrm{~ms}$; TE, ten linearly-spaced echoes from 10 to $100 \mathrm{~ms}$; array size, $256 \times 256$; field of view, $50 \mathrm{~mm}$; flip angle, $0^{\circ}$; readout bandwidth, $50 \mathrm{kHz}$; slice thickness, $2 \mathrm{~mm}$; slices, 5; average, 2; and in-plane resolution, $195 \mu \mathrm{m}$. For each set, ten images with a TR/TE ratio ranging from $1000 / 10 \mathrm{~ms}$ to $1000 / 100 \mathrm{~ms}$ were also acquired. The power and receiver gain of the scanner was kept constant for all experiments to ensure that signal scaling conditions were identical.

For $\mathrm{T}_{2}$ measurements five sets of samples from five different rats were used. In each set, one leg was shocked and the other leg from the same rat was used as a control. Following MRI measurements the tendons were removed from the explants and muscle wet weight was determined. Then the muscles were placed in a vacuum oven at $60{ }^{\circ} \mathrm{C}$ until completely dry (constant weight) in order to obtain the dry weight of the muscle. From this the water content and thus the hydration of the muscle tissue was calculated.

In the second series of experiments the whole animal was placed in the scanner. MRI measurements were carried out using a custom made Helmholtz coil with internal diameter of $25 \mathrm{~mm}$. MR imaging was performed as previously described with the following changes in parameters. The array size is $128 \times 128$ and ten slices were prescribed, $2 \mathrm{~mm}$ thick, and separated by $2 \mathrm{~mm}$ over the entire mid-thigh region of the leg. Typically, one imaging sequence took 4.5 minutes to complete. Imaging began at approximately 1 hour after shock and continued until 3 hours post shock. 


\section{ASESSING THE MEMBRANE DAMAGE}

\section{Ex vivo MRI Results}

In the first series of experiments the animals were shocked, left for 3 hours before intact SOL and EDL muscles, as well as a part of the BFM, were harvested. The muscles were placed in sealed cuvettes for MR imaging. FIGURE 2A illustrates how the samples were placed in the magnet for MRI measurements and FIGURE 2B illustrates a cross-sectional view of samples inside the coil.

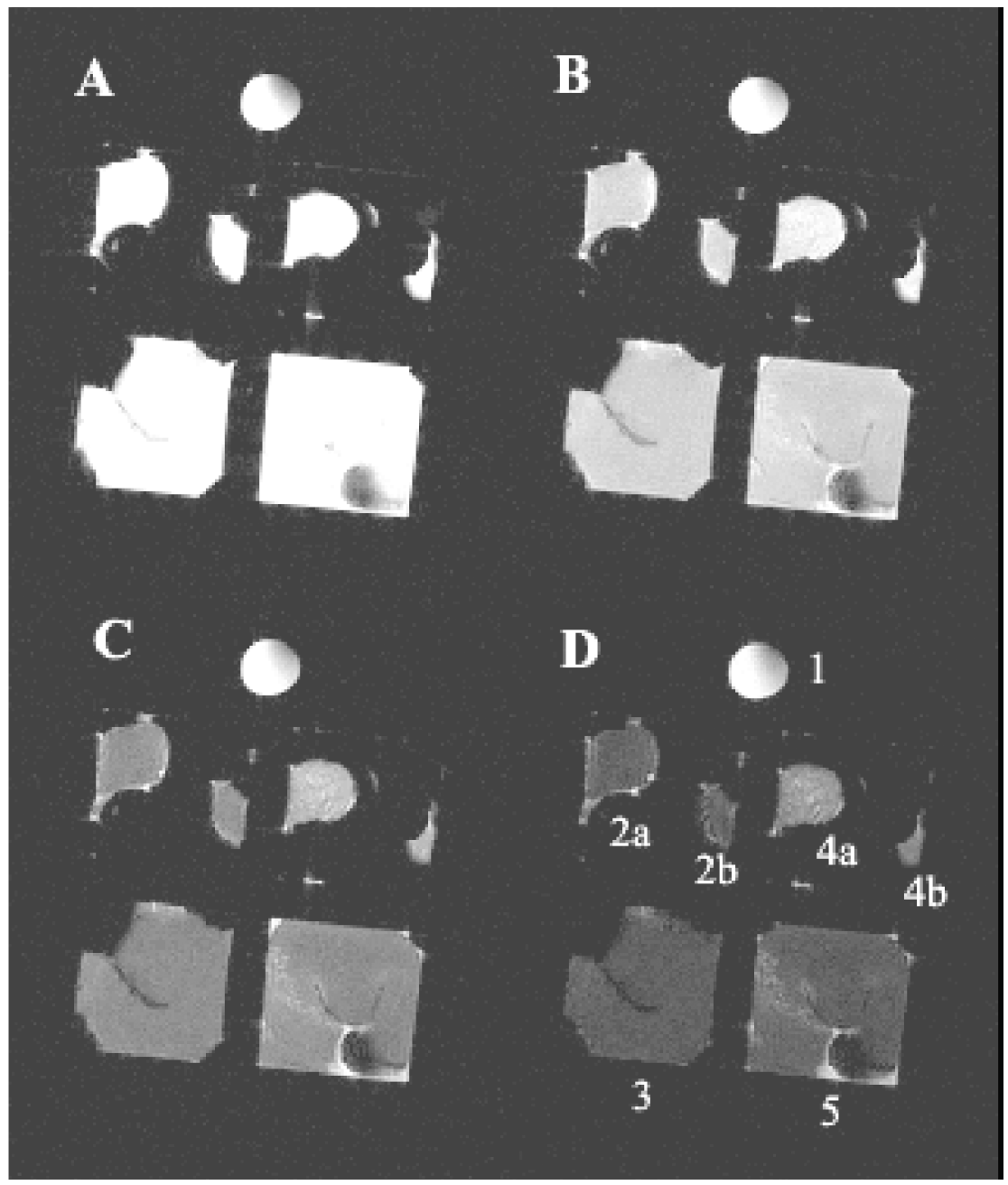

FIGURE 3. Illustration of $T_{2}$-weighted $M R$ images. The animal was anaesthetized and shocked. Three hours post shock the animal was sacrificed and SOL, EDL, and BFM were harvested and placed in cuvettes for imaging. Images were acquired at echo times (TE) ranging from 10 to $100 \mathrm{~ms}$. Four sets of images are shown: (A) TE $=15 \mathrm{~ms}$; (B) TE $=30 \mathrm{~ms}$; (C) $\mathrm{TE}=60 \mathrm{~ms}$; and (D) TE $=90 \mathrm{~ms}$. The numbering scheme (1-5) indicated in panel $\mathbf{D}$ is the same as in FIgURE 1. The labels $\mathbf{a}$ and $\mathbf{b}$ represent SOL and EDL muscles, respectively. 
A.
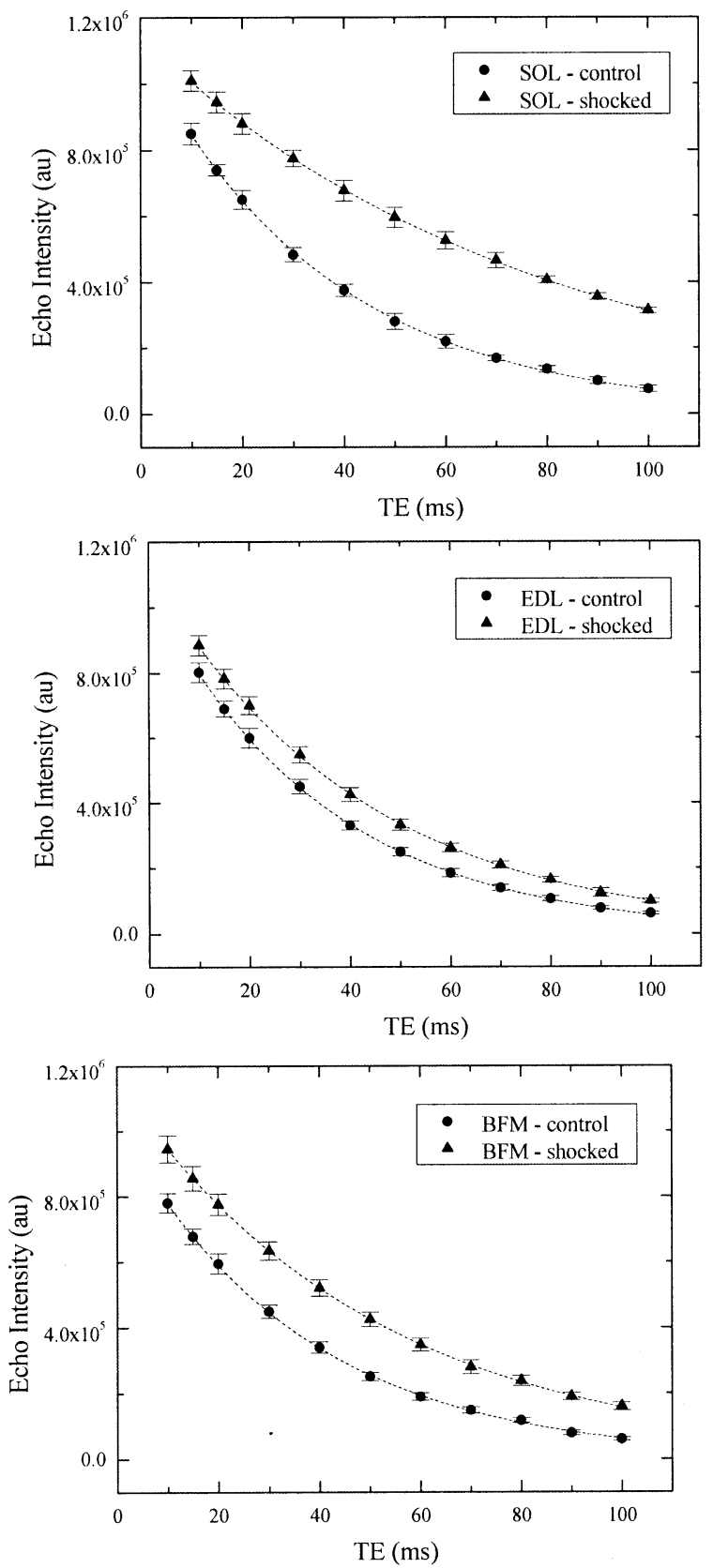

FIGURE 4. The dependence of MR signal intensities on the echo time for normal and electroporated rat muscles. A: The data were fitted to an exponential decay curve of the signal amplitude as a function of echo time, as shown by dashed lines. Values of $\mathrm{T}_{2}$ obtained from these plots are shown in TABLE 1. 
B.
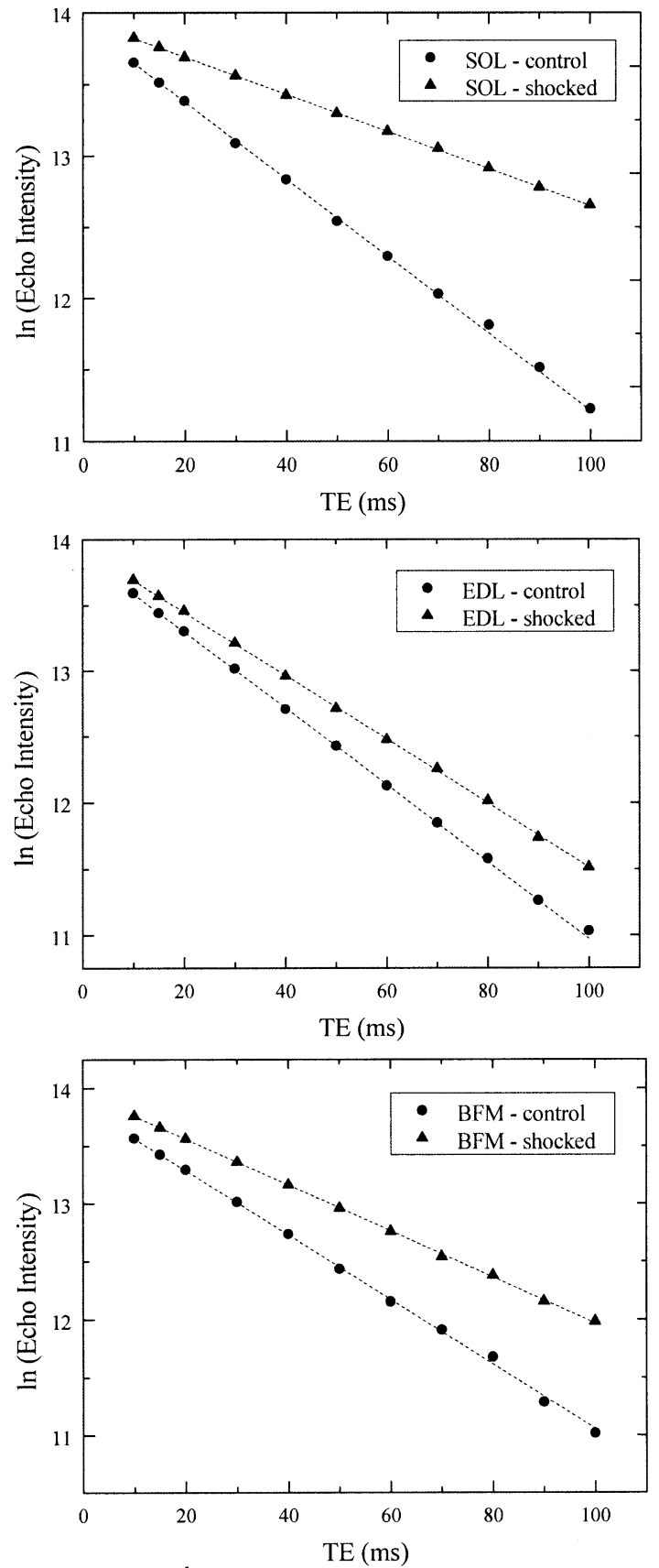

FIGURE 4-continued. B: Logarithmic plots of the dependence of MR signal intensities on the echo time. Mean values \pm SD are shown; $n=4-5$ animals. 
FIGURE 3 shows a set of $T_{2}$ weighted images at echo times ranging from 15 to $90 \mathrm{~ms}$. As seen from the figure, signal intensity decreases as TE increases; however, it is also clear that the contrast between the shocked and the untreated muscles increases with increasing TE.

FIGURE 4A shows the dependence of MR intensities on the echo time for normal and electroporated rat muscles. The data were fitted to an exponential decay curve of the signal amplitude as a function of echo time. It is clear that echo intensity is higher in the shocked muscles at all echo times with the largest difference in SOL and the smallest in EDL. FIGURE 4B shows the log transformation of the data. The slope of each curve represents the expected value of the $T_{2}$ relaxation time. $T_{2}$ values of control and shocked muscle samples as measured by MRI are listed in TABLE 1. Shocking significantly increased $\mathrm{T}_{2}$ in all muscles $(P<0.006)$. The largest increase $(108 \%)$ was observed in SOL. In EDL shocking increased $\mathrm{T}_{2}$ by $20 \%$ and finally in $\mathrm{BFM} \mathrm{T}_{2}$ was increased by $40 \%$.

\section{Muscle Water Content}

The hydration of the muscle tissue was measured by determining the water content. As shown in TABLE 1, muscle water content was significantly increased in all shocked muscles compared to untreated controls. In SOL water content was increased by $15 \%$, whereas in EDL and BFM the increase was $4 \%$.

\section{In vivo MRI Results}

In order to investigate the development of the changes in $T_{2}, M R$ imaging was performed in vivo starting approximately 1 hour after shock and continuing until approximately 3 hours after. FIGURE 5A shows two images of the mid-thigh region of a shocked hind leg from a live rat. The first image is recorded 63 min post shock and the other at 184 min post shock. From these images it can be observed how the signal

TABLE 1. Spin-spin relaxation time $\left(T_{2}\right)$ and water content of three different rat muscles following electroporation in vivo

\begin{tabular}{lclcc}
\hline \multicolumn{1}{c}{ Sample $^{a}$} & \multicolumn{1}{c}{$\mathrm{T}_{2}(\mathrm{~ms})^{b}$} & $P$ Value & Hydration & $P$ Value \\
\hline SOL (control) & $36.90 \pm 1.43(5)$ & & $0.735 \pm 0.022(5)$ & \\
SOL (shocked) & $76.80 \pm 3.07(5)$ & 0.00002 & $0.845 \pm 0.013(5)$ & 0.0005 \\
EDL (control) & $34.41 \pm 1.41(5)$ & & $0.727 \pm 0.013(5)$ & \\
EDL (shocked) & $41.37 \pm 1.55(5)$ & 0.006 & $0.758 \pm 0.020(5)$ & 0.017 \\
BFM (control) & $36.11 \pm 0.72(4)$ & & $0.741 \pm 0.017(4)$ & \\
BFM (shocked) & $50.42 \pm 0.017(4)$ & 0.001 & $0.771 \pm 0.017(4)$ & 0.031 \\
\hline
\end{tabular}

${ }^{a}$ Samples are as follows: SOL, soleus; EDL, extensor digitorum longus; and BFM, biceps femoris muscle. For description of control and shocked muscles and determination of hydration, see text. Mean values \pm SD are shown with the number of observations in parentheses. $P$ indicates the significance of the difference between the control and the shocked muscles. Student's paired $t$ test.

${ }^{b} \mathrm{~T}_{2}$ (in millisecond)s were determined by fitting the signal amplitude from the echoes of the multi-slice multi-echo experiments to the spin-spin signal decay equation: $M_{(\mathrm{x}, \mathrm{y})}=M_{0(\mathrm{x}, \mathrm{y})} \bullet \exp$ $\left(-T E / T_{2}\right)$, where $\mathrm{M}_{0}$ is the amount of magnetization at thermal equilibrium, TE is the experimental echo time, and $\mathrm{T}_{2}$ is the spin-spin relaxation time. 
A

a

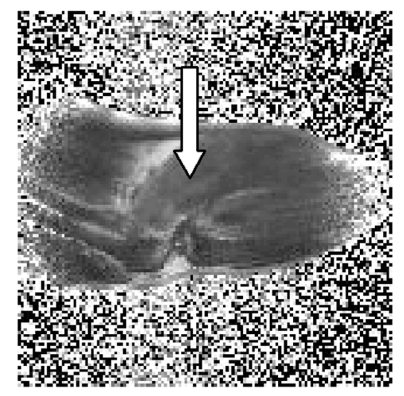

b

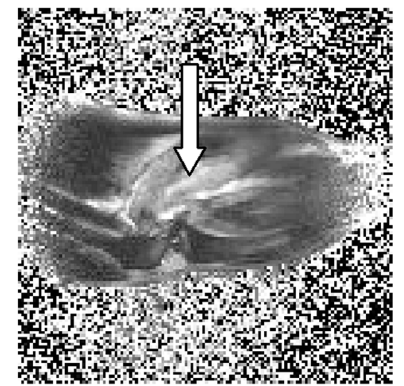

B

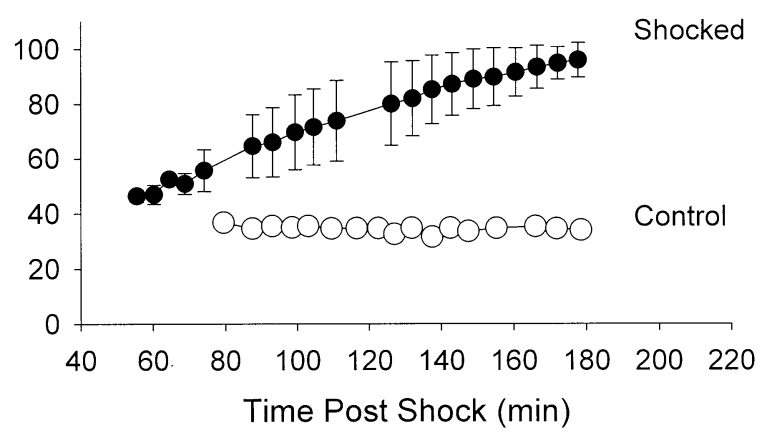

FIGURE 5. A: Two images of the mid-thigh region of a live rat ( $a$ recorded 63 min post shock, $b$ recorded 184 min post shock). The region used for calculation of mean $\mathrm{T}_{2}$ is depicted by arrows $\mathbf{B}$ : Mean $\mathrm{T}_{2}$ values for shocked (solid circles, $\mathrm{SD}, n=25$ pixels) and unshocked controls (open circles, $n=25$ pixels).

intensity increases with time. The signal intensities at the different TE times were used to fit an exponential decay and measure the $T_{2}$ value in each voxel. $T_{2}$ values were calculated from the images of each animal for a region of interest $(\mathrm{ROI}=$ 25 pixels) and the average value as a function of time is shown in FIgURE 5B. As seen from this figure, $\mathrm{T}_{2}$ increases progressively to about $180 \%$ over a period of 180 min after shock.

\section{Histology}

Muscle tissue samples were taken from the electrically injured rat hind limb after applying a different number of pulses to obtain histologic dose-response data. A muscle biopsy was performed at the end of a neurophysiology study (6 hours post shock) and the tissue was fixed in $10 \%$ formalin. The samples were paraffin-embedded, cut, slide-mounted, and hematoxylin and eosin-stained (H\&E). 


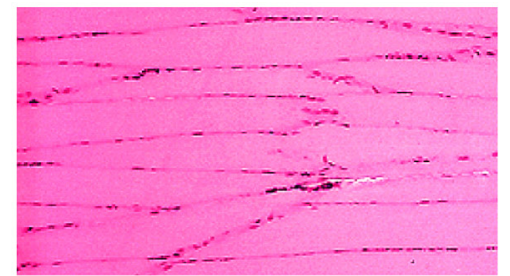

No-shock control

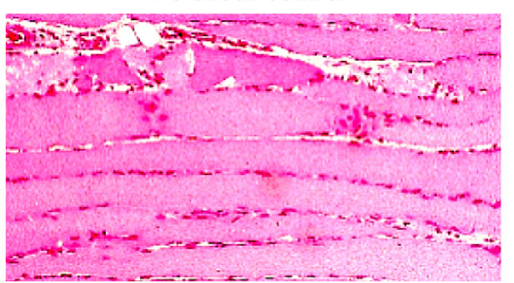

Six current pulses

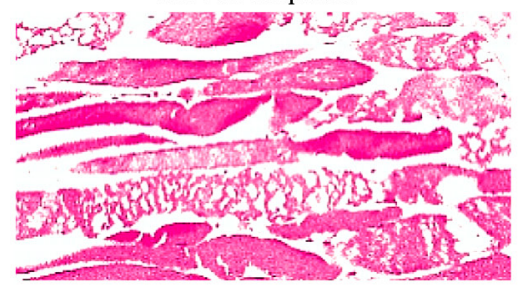

Twelve Shock Pulses

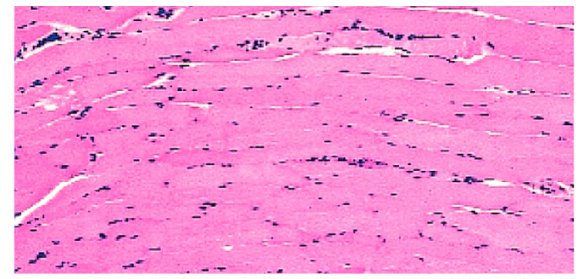

Three current pulses

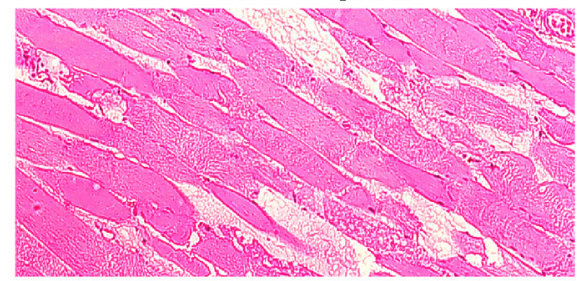

Nine Current Pulses

FIGURE 6. H\&E stain of histologic sections of hind limb muscle. Muscle has been subjected to multiple DC electric current pulses ( $2 \mathrm{~A}, 4 \mathrm{~ms}, 10$-s interval) or no shock control. Images shown were selected from the subsets exposed to 0 (control), 3, 6, 9, and 12 electric shock pulses. Muscle biopsy taken 6 h post shock. (Original magnification $\times 150$.)

A histologic examination of formalin-fixed paraffin-embedded sections of the shocked muscle is displayed in FIGURE 6. This reveals extensive vacuolization, which suggests increased water within damaged myocytes. One can also observe areas of sarcoplasm relatively devoid of eosinophilic contractile proteins and hypercontraction band degeneration, which was not seen in the muscle from the unshocked limb. These results suggest that non-thermal electrical effects can induce structural alteration of the cell.

\section{The Increase of the Hydrophobic Exposure in Injured Cells}

The damage of the cell membranes increases the hydrophobic exposure to surrounding water, which marks the magnetic relaxation of this water. The increase of hydrophobic exposure (and, implicitly, the degree of structural alteration of the cells) can be estimated based on a statistical mechanics approach which interprets the $T_{2}$-MRI data in terms of dynamical changes and recompartmentalization of water after injury. ${ }^{12}$ The net structural change (denaturation) of the cellular components in each muscle tissue is given by the value of $\alpha(0 \leq \alpha \leq 1)$, which is a measure of the 
TABLE 2. Predicted structural alteration in three different skeletal muscles due to electroporation

\begin{tabular}{ccccc}
\hline Sample $^{a}$ & $\begin{array}{c}\rho_{c} \\
\text { (control) }\end{array}$ & $\begin{array}{c}T_{2}{ }^{(h y d)} \\
\text { (shocked) }\end{array}$ & $\begin{array}{c}T_{2}{ }^{(i)}-\mathrm{T}_{2}{ }^{(h y d)} \\
\text { (shocked) }\end{array}$ & $\begin{array}{c}\alpha \\
\text { (shocked) }\end{array}$ \\
\hline SOL & 0.683 & $71.58 \mathrm{~ms}$ & $5.22 \mathrm{~ms}$ & 0.08 \\
EDL & 0.673 & $40.4 \mathrm{~ms}$ & $0.97 \mathrm{~ms}$ & 0.03 \\
BFM & 0.665 & $42.49 \mathrm{~ms}$ & $7.93 \mathrm{~ms}$ & 0.18 \\
\hline
\end{tabular}

${ }^{a}$ Samples are as follows: SOL, soleus; EDL, extensor digitorum longus; and BFM, biceps femoris muscle. For description of control and shocked muscles, see text.

increase of hydrophobic exposure after injury ( $\alpha=0$, for healthy muscle, and $\alpha=1$, for the extreme case of denaturated cells). This is extracted by a numerical computation from the difference between values for injured and non-injured muscles. Thus, by replacing $\eta_{l}$ for shocked muscles (TABLE 1) in the Zimmerman-Brittin eqs., ${ }^{12}$ we obtain $T_{2}\left(\right.$ hyd) values (TABLE 2 ) which are typically smaller than the measured $\mathrm{T}_{2}$. This means that there is also an intrinsic contribution to the magnetic relaxation of water in injured muscles $\left(T_{2}{ }^{(i)}-T_{2}{ }^{(h y d)}\right)$, which corresponds to the alteration of the dynamics of water in contact with disrupted cell membranes and denaturated proteins, as we described above. Under such circumstances, we used the measured magnetic relaxation times $T_{2}{ }^{(i)}$ (TABLE 1 ) and hydration values $\eta_{l}$ (TABLE 1 ) in the above equations to extract the corresponding values $\alpha^{(\mathrm{SOL})}, \alpha^{(\mathrm{EDL})}$, and $\alpha^{(\mathrm{BFM})}$. They predict the degree of alteration of the cellular components following electroporation. Precisely, $\alpha$ represents the fraction of the exposed hydrophobic parts of the cellular components affected by electroporation. Our predictions for $\alpha^{(\mathrm{SOL})}, \alpha^{(\mathrm{EDL})}$, and $\alpha^{(\mathrm{BFM})}$ are displayed in TABLE 2 .

\section{DISCUSSION}

When muscle cells are electroporated in vivo, a progressive alteration of the state of water in the tissue is observed (FIG. 5B). Electroporation may lead to the formation of edema due to osmotic water inflow into the muscle tissue. Edema raises the local hydration level over its physiological value. In the absence of significant structural modifications, the amount of immobilized water as well as water in the first hydration layer remains unchanged. ${ }^{12}$ The accumulated water adds extra hydration layers to the cellular components and changes the fractions of the spin population. This, in turn, increases the MR signal.

A secondary effect which can influence the long-term pattern of $\mathrm{T}_{2}$ is the development of secondary structural damage. Upon electroporation of the cell membrane, water can invade membrane compartments that are usually separated from excess amounts of water. This irregular hydration of the cellular membrane may increase the permeability of the membrane leading to influx of $\mathrm{Ca}^{2+}$, thus accelerating tissue damage due to activation of degradative processes intrinsic to the muscle cell. ${ }^{6,8-10}$ An increase of the structural damage affects not only the compartmentalization of the spin population, but also modifies spin dynamics during magnetic relaxation, that is, the exposure of the hydrophobic layer of the damaged membrane changes the 
dynamics of the vicinal water. ${ }^{12}$ In this context, the increase in the MRI contrast observed in the injured tissue can be interpreted as a superposition of edema and structural damage at the molecular level.

In this study three different muscles were tested. The muscles differ from each other with regards to the composition of fiber type. SOL is mainly composed of slow-twitch fibers whereas EDL is mainly composed of fast-twitch fibers. ${ }^{21}$ No data on fiber type composition was found for BFM. Not surprisingly we find that the muscles respond differently to shocking. However, an interesting aspect of the results is that there is no fixed relation between the degree of edema and the amount of structural damage. SOL clearly experiences the largest degree of edema (15\% increase), whereas BFM experiences the largest degree of structural changes (18\%). The reason for this difference is not clear; however, anatomic differences may be important. First, the amount of current passing through the tissue depends on the resistance of that tissue, and we would expect this to vary at different locations in the leg. Vasculature and the tightness of the facia surrounding the muscles will be important in determining the degree of edema developing in the muscle. Also, since the electric field is along the length of the fibers, fiber length may be important for the susceptibility to damage. Finally, one might expect that one fiber type is more sensible to electroporation damage than the other. However, in contrast to the findings in this study it has been shown that when isolated muscles are electroporated ex vivo (with the electric field perpendicular to the length of the fiber), EDL is more easily damaged than soleus. ${ }^{7}$

The present approach sheds light on the mechanism of development of the electrical trauma and suggests a way to discriminate between the two main contributions to the MRI contrast, edema and the state of integrity of the cellular components.

The major conclusion that can be drawn from this study is that MRI can be used as an efficient non-invasive method for evaluation of muscle electrical trauma. However, the increase in $\mathrm{T}_{2}$ has two components and it is not necessarily the area showing the largest increase in the water content (edema) which suffers the most structural damage. Future studies will allow us to test the predictions on the structural damage and to locate the damaged components.

\section{ACKNOWLEDGMENTS}

The research presented here has been supported by the National Institutes of Health Grant R01 GM61101 and the Danish Medical Research Council Grant 22-02-0523.

\section{REFERENCES}

1. Hunt, J.L., R.M. Sato \& C. BaXter. 1980. Acute electric burns: current diagnostic and therapeutic approaches to management. Arch. Surg. 115: 434-438.

2. Lee, R.C., D.C. GaYlor, D. Bhatt \& D.A. IsRael. 1988. Role of cell membrane rupture in the pathogenesis of electrical trauma. J. Surg. Res. 44: 709-719.

3. Chilbert, M.A. 1992. High-voltage and high-current injuries. In Electrical Stimulation and Electropathology. J. Patrick Reilly et al. Cambridge University Press. Cambridge, UK.

4. LEE, R.C. 1997. Injury by electrical forces: pathophysiology, manifestations, and therapy. Curr. Prob. Surg. 34: 677-765. 
5. GeHL, J. 2003. Electroporation: theory and methods, perspectives for drug delivery, gene therapy and research. Acta Physiol. Scand. 177: 437-447.

6. Gissel. H. \& T. Clausen. 2003. $\mathrm{Ca}^{2+}$ uptake and cellular integrity in rat EDL muscle exposed to electrostimulation, electroporation, or A23187. Am. J. Physiol. Regul. Integr. Physiol. 285: R132-R142.

7. Clausen, T. \& H. Gissel. 2005. Role of $\mathrm{Na}^{+}, \mathrm{K}^{+}$pumps in restoring contractility following loss of cell membrane integrity in rat skeletal muscle. Acta Physiol. Scand. 183: 263-271.

8. Publicover, S.J., C.J. Duncan \& J.L. Smith. 1978. The use of A23187 to demonstrate the role of intracellular calcium in causing ultrastructural damage in mammalian muscle. J. Neuropathol. Exp. Neurol. 37: 554-557.

9. JACKSON, M.J., D.A. JonES \& R.H. EDwARDS. 1984. Experimental skeletal muscle damage: the nature of the calcium-activated degenerative processes. Eur. J. Clin. Invest. 14: 369-374.

10. Armstrong, R.B., G.L. Warren \& J.A. Warren. 1991. Mechanisms of exerciseinduced muscle fibre injury. Sports Med. 12: 184-207.

11. Block, T.A., J.N. Aarsvold, K.L. Mathews, II, et al. 1995. Nonthermally mediated muscle injury and necrosis in electrical trauma. J. Burn Care \& Rehabil. 16: 581588.

12. Despa, F., R.S. BERRY \& R.C. LEe. 2005. Linking $T_{2}$-MRI to protein unfolding. Submitted for publication.

13. Denisov, V.B. \& B. Halle. 1998. Thermal denaturation of ribonuclease A characterized by water ${ }^{17} \mathrm{O}$ and ${ }^{2} \mathrm{H}$ magnetic relaxation dispersion. Biochemistry 37: 95959604.

14. Denisov, V.P., B.H. Jonsson \& B. Halle. 1999. Hydration of denatured and molten globule proteins. Nat. Struct. Biol. 6: 253-260.

15. Bertram, H.C., A.H. Karlsson, M. Rasmussen, et al. 2001. Origin of multiexponential T(2) relaxation in muscle myowater. J. Agric. Food Chem. 49: 3092-3100.

16. Karczmar, G.S., L.P. River, J. River, et al. 1994. Prospects for assessment of the effects of electrical injury by magnetic resonance. Ann. N.Y. Acad. Sci. 720: 176180.

17. Fleckenstein, J.L., D.P. Chason, F.J. Bonte, et al. 1995. High-voltage electric injury: assessment of muscle viability with MR imaging and Tc-99m pyrophosphate scintigraphy. Radiology 195: 205-210.

18. Nettelblad, H., K.A. Thuomas \& F. SJoberg. 1996. Magnetic resonance imaging: a new diagnostic aid in the care of high-voltage electrical burns. Burns 22: 117-119.

19. Ohashi, M., J. Kolzumi, Y. Hosoda, et al. 1998. Correlation between magnetic resonance imaging and histopathology of an amputated forearm after an electrical injury. Burns 24: 362-368.

20. Hannig, J., D.A. Kovar, G.S. Abramov, et al. 1999. Contrast enhanced MRI of electroporation injury [abstract]. Proceedings of the 1st Joint BMES/EMBS Conference, CD \#1078.

21. Deschenes, M.R., A.A. BritT \& W.C. Chandler. 2000. A comparison of the effects of unloading in young adult and aged skeletal muscle. Med. Sci. Sports Exerc. 33: $1477-1483$. 